\title{
Airplane positioning using airborne collision avoidance system data
}

\author{
Volodymur Kharchenko ${ }^{1}$, Ivan Ostroumov ${ }^{1, *}$, Nataliia Kuzmenko $^{1}$ and Arkadiy Larionov ${ }^{2}$ \\ ${ }^{1}$ National Aviation University, Kyiv, Ukraine \\ ${ }^{2}$ Moscow State University of Civil Engineering, 26 Yaroslavskoye Shosse, Moscow, 109377, Russia
}

\begin{abstract}
Mid-air collision is an important problem of modern air transport system. Development of Airborne Collision Avoidance System (ACAS) is grounded on safety criteria in order to support air traffic capacity and equipment. In research, we study the possibility and performance of airplane positioning by data accumulated in ACAS. ACAS surveillance equipment supports accurate range and poor angular measurements of airspace user's data. An approach is based on a fusion of surveillance data with airplanes locations obtained from decoded Automatic Depended Surveillance-Broadcast messages. Performance of positioning approach is considered in more detail. An error of positioning in the horizontal plane is estimated in relation with uncertainty of airspace users' location. Numerical demonstration with a live air traffic data indicates poor positioning accuracy in comparison with primary positioning system on-board of airplane and accuracy dependence from geometry and capacity of air traffic.
\end{abstract}

\section{Introduction}

An Airborne Collision Avoidance System (ACAS) is an automated air traffic monitoring system with the aim of finding potential conflicts between air space users in the air transport system and issuing pilot recommendations for their solution [1]. A safe solution of mid-air collisions is a primary task of an intelligent transport system [2]. ACAS surveillance equipment operates similarly to secondary surveillance radars, providing accurate measurement of ranges to other airspace users. According to that, ACAS can be considered as an on-board sensor for measuring ranges to air traffic.

ACAS has been approved by numerous normative documents at the international level [1, 3]. ACAS II is a mandatory system for heavy airplanes [3], and ACAS I is included to the minimum equipment list of general aviation in many regions. ACAS has been standardized in three modifications, but today, only two of them are implemented $[3,4]$ :

- $\quad$ ACAS I informs a pilot about air traffic around (TA - Traffic Advisory);

- $\quad$ ACAS II provides observation of surrounding air traffic and visualization on TA display. ACAS II detects mid-air collision and generates a coordinated Resolution Advisory (RA) for pilots to avoiding reducing a safe separation minimum.

\footnotetext{
*Corresponding author: ostroumovv@ukr.net
} 
ACAS grounds on independent surveillance for air traffic detection using direction finder equipment [4]. ACAS includes a computing unit for interrogation generation at a frequency of $1030 \mathrm{MHz}$, similar to interrogation signals of a secondary surveillance radar and transmits them through antenna system. The airplane transponder receives interrogation and automatically generates a response at $1090 \mathrm{MHz}$. ACAS receives the responses and estimates the range to the airspace user, by the time of arrival method.

Directional antenna of ACAS supports radio bearing measurement to airspace users with poor accuracy, which depends on the geometry of airplanes' locations. In common, the accuracy of bearing measurements does not exceed $3^{\circ}$, but in particular cases, an error can reach $30^{\circ}$. Valuable error of angular data makes unavailable Angle of Arrival method for positioning. Unavailability of precise mutual airplanes location makes impossible to resolve the conflict by using a coordinated maneuver in the horizontal plane, which is a major peril for ACAS III development [1].

The reply signal from the airplane transponder contains information about flight number, call sign and barometric altitude that is used by ACAS operation algorithm to detect a mid-air collision.

Disadvantages of ACAS II and the constant increase of airspace capacity combined with the problems of integrating unmanned aerial vehicles in controlled air traffic [5] have led to finding ways to improve ACAS standard. The proposed ACAS X concept is based on the probabilistic approach with the use of dynamic programming for choosing the optimal maneuvering path $[1,6,7]$. Surveillance of ACAS X is improved by using data from the Automatic Dependent Surveillance-Broadcast (ADS-B). ACAS X utilizes the next models [1]:

- ACAS Xa - designed to replace ACAS II using an active request and passive surveillance by ADS-B;

- ACAC Xo - extended version of ACAS Xa for use in congested airspace;

- ACAS Xu - version for use at Remotely-Piloted Aircraft System;

- ACAS Xp - a version for use by general aviation grounded on ADS-B surveillance.

Future ACAS X development will be entirely based on the widespread use of ADS-B data, which includes extended information about airspace user's locations [8, 9].

Precise location detection is a key element of airplane navigation in order to keep air transport system at required safety level. A modern airplane utilizes various positioning equipment in order to guarantee a required level of data continuity and integrity. Numerous advantages of Global Navigation and Satellite System (GNSS) make usage of GNSS as a primary positioning sensor, but some factors such as poor geometry of satellite segment, delays of radio signals in ionosphere and troposphere, interference [10], unintentional jamming [11, 12], or simple equipment malfunction [13] can reduce positioning performance and may lead to GNSS receiver lock. Statistic of aviation incidents indicates 24 malfunctions of on-board GNSS during 2017 [14]. In case of GNSS positioning lock, inertial navigation system can be used. However, the time of its usage is limited due to additive error. Also, the data from a pair of navigational aids can be used in Flight Management System (FMS) for airplane positioning. In this case, FMS detects effective pair of navigational aids [15] and tunes Distance Measuring Equipment and VHF omnidirectional ranging (VOR) equipment to perform measurement of navigational data for airplane coordinates detection. Positioning by navigational aids requires a wide network of ground equipment. Also, a performance of positioning depends on the geometry of ground network and can not support specifications for area navigation less than 1 NM (RNAV 1), which will be introduced in the near future [16]. Thus, a problem of alternative or stand-by positioning equipment developing is important today. 
The main objective of the paper is an investigation of the capability of ACAS X surveillance and communication data usage for airplane positioning. Performance analysis of positioning by ADS-B data also needs to be studied to detect the influence of uncertainties of airspace users' location into the result of positioning in accordance with performance-based navigation regulation [17]. The preliminary study of alternative positioning, navigation and timing approaches indicates positioning error dependence from various factors that significantly limits positioning performance [18, 19]. Positioning by ACAS data makes positioning accuracy dependent on only from air traffic capabilities that provide positioning independence from ground facilities.

\section{Method of positioning}

According to ACAS X development, air traffic surveillance will be provided by a combination of passive surveillance by ADS-B and active one, by mode "S" interrogation. According to that, information from both systems can be combined to determine airplane location.

Let's consider the case of ACAS interaction with two airspace users. It should be noted that ACAS I and ACAS II positioning algorithms are the same and are based on cooperation with communication mode "S" of airplane transponders. In this case, ACAS generates request messages and scans the airspace with the help of a directional antenna. Mode "S" transponders within ACAS operational area receive the request signals and transmit reply signals. On the other hand, each airspace user is equipped with ADS-B transponder that continuously transmits its location in airspace. Therefore, each airspace user can be represented as a navigation point with known coordinates of location and known ranges to them obtained by ACAS measurements (fig 1).

The solution of navigation equation, depending on the amount of available data, can be obtained either in the global Earth-Centered-Earth-Fixed (ECEF) or in local NorthEast-Down/Up (NED/NEU) Cartesian reference frame. Accuracy of positioning in horizontal and vertical planes is an important task of performance estimation. Therefore, it is advisable to perform a solution of the navigation problem in the local coordinate system to obtain the initial matrices for the accuracy estimation. Also, in order to simplify the calculation in a local reference frame, we use the previous coordinates of airplane location and its speed vector for prediction of airplane location at the time of measurement by dead-reckoning method or by simple sequential analysis.

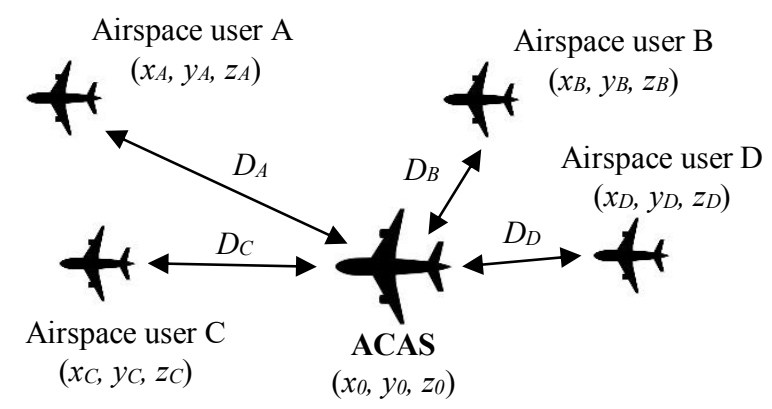

Fig. 1. Positioning by data from airspace users.

After converting the initial data to the local Cartesian coordinate system, the system of navigation equations can be represented as follows:

$$
D^{2}=\left(x_{0}-x_{A C F T i}\right)^{2}+\left(y_{0}-y_{A C F T i}\right)^{2}+\left(z_{0}-z_{A C F T i}\right)^{2}, i=[1, n],
$$


where $n$ is a number of visible airspace users; $x_{A C F T i}, y_{A C F T i}, z_{A C F T i}$ are coordinates of $i^{\text {th }}$ airspace user; $x_{0}, y_{0}, z_{0}$ are coordinates of airplane; $D_{i}$ is a distance between airplane and airspace users.

Solutions of (1) will be coordinates $\left(x_{0}, y_{0}, z_{0}\right)$ of ACAS equipped airplane. In case, of two airspace users, the altitude data is not taken into account in (1) and, as a result, we have two solutions. The correct solution will be chosen in the proximity of the location to the previous position.

A solution of nonlinear navigation equations (1) can be obtained by linearization with a help of Taylor series expansion by first order of derivatives with the use of the leastsquares method [20].

An iterative searching algorithm uses start point $\left(x_{\text {ref, }} y_{\text {ref, }} z_{\text {ref }}\right)$ to estimate differences in coordinates between airplane location and reference point. The difference between the true values of distance and the values on a given iteration can be represented as a Taylor series expansion by derivatives of the first order:

$$
\Delta D=\frac{x_{r e f}-x_{A C F T}}{D_{r e f}} \Delta x+\frac{y_{r e f}-y_{A C F T}}{D_{r e f}} \Delta y+\frac{z_{r e f}-z_{A C F T}}{D_{r e f}} \Delta z
$$

Equation (2) can be represented in the matrix form:

$$
\triangle D_{A C A S}=H_{A C A S} \triangle u
$$

where

$$
\begin{gathered}
\Delta u=\left[\begin{array}{c}
\Delta x \\
\Delta y \\
\Delta z
\end{array}\right], \\
\Delta D_{A C A S}=\left[\begin{array}{c}
D_{r e f 1}-D_{A C A S 1} \\
D_{r e f 2}-D_{A C A S 2} \\
D_{r e f 3}-D_{A C A S 3}
\end{array}\right], \\
H_{A C A S}=\left[\begin{array}{ccc}
\frac{x_{r e f}-x_{A C F T 1}}{D_{r e f 1}} & \frac{y_{r e f}-y_{A C F T 1}}{D_{r e f 1}} & \frac{z_{r e f}-z_{A C F T 1}}{D_{r e f 1}} \\
\frac{x_{r e f}-x_{A C F T 2}}{D_{r e f 2}} & \frac{y_{r e f}-y_{A C F T 2}}{D_{r e f 2}} & \frac{z_{r e f}-z_{A C F T 2}}{D_{r e f 2}} \\
\frac{x_{r e f}-x_{A C F T 3}}{D_{r e f 3}} & \frac{y_{r e f}-y_{A C F T 3}}{D_{r e f 3}} & \frac{z_{r e f}-z_{A C F T 3}}{D_{r e f 3}}
\end{array}\right] .
\end{gathered}
$$

The solution of (3) is obtained by the least-squares method:

$$
\Delta u=\left(H^{T} H\right)^{-1} H^{T} \Delta D
$$

The $\Delta u$ value represents the accuracy at each iteration step, which approximates the point $\left(x_{r e f}, y_{r e f}, z_{r e f}\right)$ to the true value of airplane location with a predefined accuracy.

In general case, positioning method by ACAS X data can be represented as a scheme in fig. 2. According to ACAS specification [1, 4], ACAS X provides distance measurements to airspace users and receives ADS-B messages. All slant distances in ACAS should be transformed to horizontal component in the reference frame, and air traffic location also should be transformed from the geocentric Latitude Longitude 
Altitude (LLA) form to the local one (e.g. NEU). A reference point of the local frame is placed in the coordinates of the previous airplane location. Transformed coordinates of navigation points are used to construct a matrix of partial derivatives $H_{A C A S}$, which is used by an iterative algorithm to find a solution with a certain accuracy by (4). The center of reference frame is used as a start point of an iterative searching algorithm.

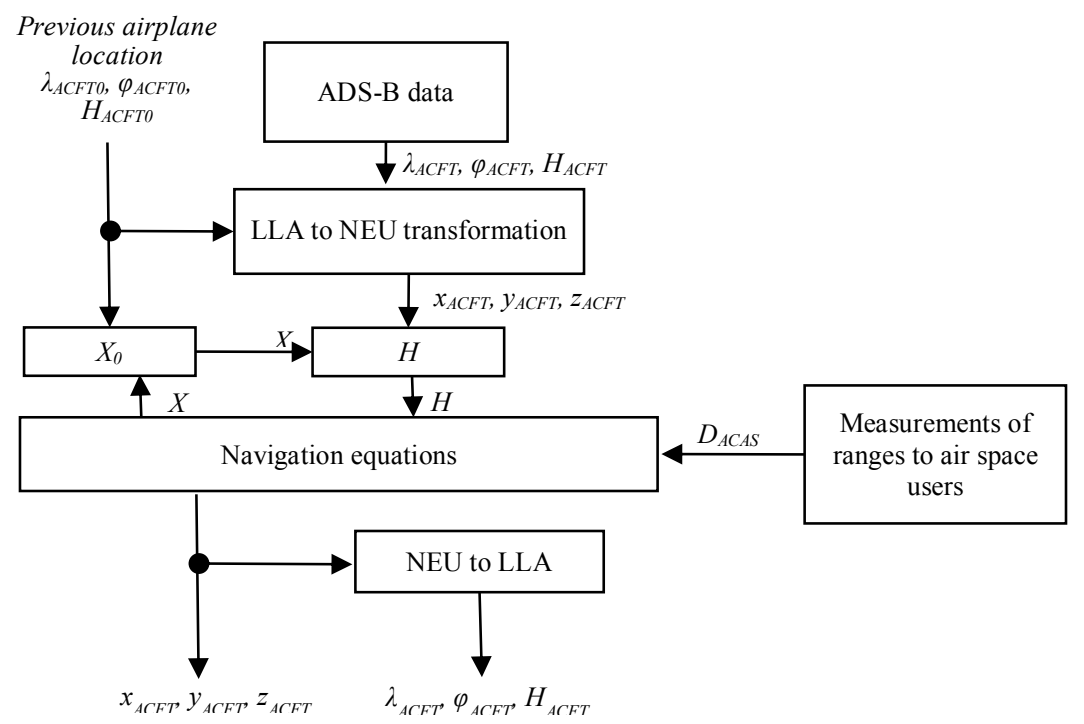

Fig. 2. Structural scheme of positioning by ACAS data.

At the end obtained coordinates of an airplane will be transformed into the global coordinate system such as LLA for sharing with other on-board equipment.

\section{Positioning performance}

Accuracy of positioning by ACAS X data depends on the geometry of air traffic distribution $\left(\varepsilon_{\mathrm{g}}\right)$, an error of measurement equipment $\left(\varepsilon_{\mathrm{ACAS}}\right)$ and accuracy of ADS-B data $\left(\varepsilon_{\mathrm{ADS}-\mathrm{B}}\right)$. Uncertainty area of airplane location can be represented as an ellipse of errors. Since ADS-B data is usually obtained from GNSS, and GNSS errors in a small area of airspace can be considered the same, ellipses of errors will have approximately the same geometric dimensions and the same characteristics in space. Uncertainty area of positioning is the result of influence of both geometry of the mutual location and the characteristics of the ellipses of errors.

In the general case, measured distance in ACAS can be represented as a component of the true distance and three error components:

$$
f(D+\Delta D)=f(D)+\varepsilon_{g}+\varepsilon_{A D S-B}+\varepsilon_{A C A S}
$$

Influence of geometry on the accuracy of coordinate information can be represented in the form of the expansion of (1) in the Taylor series with the corresponding variables:

$$
\varepsilon_{g}=\frac{d f(D)}{d x} \Delta x+\frac{d f(D)}{d y} \Delta y+\frac{d f(D)}{d z} \Delta z
$$

where $\Delta x, \Delta y, \Delta z$ are positioning errors by coordinates in a local reference frame. 
The effect of positioning errors in ADS-B data can be represented as Taylor series with the corresponding variables in the same way to (6):

$$
\varepsilon_{A D S-B}=\frac{d f(D)}{d x_{A D S-B}} \Delta x_{A D S-B}+\frac{d f(D)}{d y_{A D S-B}} \Delta y_{A D S-B}+\frac{d f(D)}{d z_{A D S-B}} \Delta z_{A D S-B}
$$

where $\Delta x_{\mathrm{ADS}-\mathrm{B}}, \Delta y_{\mathrm{ADS}-\mathrm{B}}, \Delta z_{\mathrm{ADS}-\mathrm{B}}$ are positioning errors of navigation points obtained from ADS-B messages.

Substituting (6) and (7) in (5) in the matrix form for $n$ navigation points, we will have:

$$
\triangle D=H^{T} W \Delta+H^{T}{ }_{A D S-B} W \Delta_{A D S-B}
$$

where, $H$ is the matrix of partial derivatives; $\Delta$ is a matrix of errors in axes direction; $H_{A D S-B}$ is a matrix of partial derivatives by ADS-B location data; $\Delta_{\mathrm{ADS}-\mathrm{B}}$ is a ADS-B error matrix in axes direction; $W=E\left\{\varepsilon \varepsilon^{T}\right\}^{-1}$ is a inverse correlation matrix of measuring equipment errors or weight matrix.

$$
\begin{aligned}
& \Delta^{T}=[\Delta x \Delta y \Delta z] \\
& \Delta_{A D S-B}^{T}=\left[\Delta x_{A D S-B} \Delta y_{A D S-B} \Delta z_{A D S-B}\right] \text {; } \\
& H=\left[\begin{array}{ccc}
\frac{d f\left(D_{1}\right)}{d x} & \frac{d f\left(D_{1}\right)}{d y} & \frac{d f\left(D_{1}\right)}{d z} \\
\vdots & \vdots & \vdots \\
\frac{d f\left(D_{i}\right)}{d x} & \frac{d f\left(D_{i}\right)}{d y} & \frac{d f\left(D_{i}\right)}{d z}
\end{array}\right] ; \\
& H_{A D S-B}=\left[\begin{array}{ccc}
\frac{d f\left(D_{1}\right)}{d x_{A D S-B}} & \frac{d f\left(D_{1}\right)}{d y_{A D S-B}} & \frac{d f\left(D_{1}\right)}{d z_{A D S-B}} \\
\vdots & \vdots & \vdots \\
\frac{d f\left(D_{i}\right)}{d x_{A D S-B}} & \frac{d f\left(D_{i}\right)}{d y_{A D S-B}} & \frac{d f\left(D_{i}\right)}{d z_{A D S-B}}
\end{array}\right] \text {; } \\
& \Delta D=\left[\begin{array}{c}
\Delta D_{1} \\
\vdots \\
\Delta D_{i}
\end{array}\right] .
\end{aligned}
$$

Matrix of partial derivatives by coordinates $H$ will have the same form as $H_{A C A S}$, but contains $n$ rows:

$$
H=\left[\begin{array}{ccc}
\frac{x_{0}-x_{A C F T 1}}{D_{1}} & \frac{y_{0}-y_{A C F T 1}}{D_{1}} & \frac{z_{0}-z_{A C F T 1}}{D_{1}} \\
\frac{x_{0}-x_{A C F T 2}}{D_{2}} & \frac{y_{0}-y_{A C F T 2}}{D_{2}} & \frac{z_{0}-z_{A C F T 2}}{D_{2}} \\
\cdots & \cdots & \cdots \\
\frac{x_{0}-x_{A C F T n}}{D_{n}} & \frac{y_{0}-y_{A C F T n}}{D_{n}} & \frac{z_{0}-z_{A C F T n}}{D_{n}}
\end{array}\right]
$$


Matrix of partial derivatives by airplane coordinates has an opposite form to $H$ matrix:

$$
H_{A D S-B}=-H
$$

Solution of the matrix equation (8) for $\Delta$ can be obtained using the least-squares method:

$$
\Delta=\left(\left(H^{T} W H\right)^{-1} H^{T} W\right)\left(\Delta D-H^{T}{ }_{A D S-B} W \Delta_{A D S-B}\right)
$$

An error can be estimated by the co-variation matrix:

$$
\operatorname{cov}(\Delta)=E\left[\Delta \Delta^{T}\right]
$$

Substituting (9) in (10) we have:

$\operatorname{cov}(\Delta)=E\left[\left(\left(H^{T} W H\right)^{-1} H^{T} W\right)\left(\Delta D-H^{T}{ }_{A D S-B} W \Delta_{A D S-B}\right)\left(\left(\left(H^{T} W H\right)^{-1} H^{T} W\right)\left(\Delta D-H^{T}{ }_{A D S-B} W \Delta\right.\right.\right.$ $\left.A D S-B))^{T}\right]$.

Let's introduce the designation

$$
U=\left(H^{T} W H\right)^{-1} H^{T} W
$$

Then,

$$
\begin{aligned}
& \operatorname{cov}(\Delta)=E\left[U\left(\Delta D-H^{T}{ }_{A D S-B} W \triangle_{A D S-B}\right)\left(U\left(\Delta D-H^{T}{ }_{A D S-B} W{ }_{A D S-B}\right)\right)^{T}\right]= \\
& =E\left[U\left(\Delta D-H^{T}{ }_{A D S-B} W \triangle_{A D S-B}\right)\left(\Delta D-H^{T}{ }_{A D S-B} W A_{A D S-B}\right)^{T} U^{T}\right]= \\
& =U E\left[\left(\Delta D-H^{T}{ }_{A D S-B} W A_{A D S-B}\right)\left(\Delta D-H^{T}{ }_{A D S-B} W{ }_{A D S-B}\right)^{T}\right] U^{T}= \\
& =U E\left[\left(\Delta D-H^{T}{ }_{A D S-B} W \Delta_{A D S-B}\right)\left(\Delta D^{T}-H_{A D S-B} W^{T} \Delta^{T}{ }_{A D S-B}\right)\right] U^{T}= \\
& =U\left(E \left[\left(\Delta D \Delta D^{T}-\Delta D H_{A D S-B} W^{T} \Delta^{T}{ }_{A D S-B}-\Delta D^{T} H^{T}{ }_{A D S-B} W \Delta_{A D S-B}+\right.\right.\right. \\
& \left.\left.+H^{T}{ }_{A D S-B} W{ }_{A D S-B} H_{A D S-B} W^{T} \Delta^{T}{ }_{A D S-B}\right]\right) U^{T}= \\
& =U\left(E\left[\Delta D \Delta D^{T}\right]-E\left[\Delta D H_{A D S-B} W^{T} \Delta^{T}{ }_{A D S-B}\right]-E\left[\Delta D^{T} H^{T}{ }_{A D S-B} W \Delta_{A D S-B}\right]+\right. \\
& \left.+E\left[H^{T}{ }_{A D S-B} W \triangle{ }_{A D S-B} H_{A D S-B} W^{T} \Delta^{T}{ }_{A D S-B}\right]\right) U^{T} \text {. }
\end{aligned}
$$
then:

Since the errors of measuring distances and airplane coordinates are independent,

$$
\begin{aligned}
& E\left[\Delta D \Delta^{T}{ }_{A D S-B}\right] \approx 0 \\
& E\left[\Delta D^{T} \Delta A D S-B\right] \approx 0
\end{aligned}
$$

Thus,

$$
\begin{gathered}
\operatorname{cov}(\Delta u) \approx U\left(E\left[\Delta D \Delta D^{T}\right]+E\left[H^{T}{ }_{A D S-B} W \Delta_{A D S-B} H_{A D S-B} W^{T} \Delta^{T}{ }_{A D S-B}\right]\right) U^{T}, \\
\left.\operatorname{cov}(\Delta u) \approx U\left(\operatorname{cov}(\Delta D)+H^{T}{ }_{A D S-B} W \operatorname{cov}\left(\Delta_{A D S-B}\right) H_{A D S-B} W^{T}\right]\right) U^{T},
\end{gathered}
$$

where

$$
\operatorname{cov}\left(\Delta_{A D S-B}\right)=S_{A D S-B}=\left[\begin{array}{ccc}
\sigma_{x A D S-B}^{2} & 0 & 0 \\
0 & \sigma_{y A D S-B}^{2} & 0 \\
0 & 0 & \sigma_{z A D S-B}^{2}
\end{array}\right] ; \operatorname{cov}(\Delta D)=\sigma_{d} .
$$

In the general case we have:

$$
\left.G=\operatorname{cov}(\Delta)=U\left(\sigma_{d}+H_{A D S-B}^{T} W S_{A D S-B} H_{A D S-B} W^{T}\right]\right) U^{T}
$$


Positioning errors components are:

$$
G=\left[\begin{array}{ccc}
\sigma_{x}^{2} & \sigma_{x y}^{2} & \sigma_{x z}^{2} \\
\sigma_{y x}^{2} & \sigma_{y}^{2} & \sigma_{y z}^{2} \\
\sigma_{z x}^{2} & \sigma_{z y}^{2} & \sigma_{z}^{2}
\end{array}\right] .
$$

Positioning accuracy in the horizontal plane is:

$$
\sigma_{h}^{2}=\sigma_{x}^{2}+\sigma_{y}^{2}
$$

Positioning accuracy in vertical plane can be characterized by $\sigma_{z}^{2}$ value. Total positioning accuracy in horizontal and vertical planes is:

$$
\sigma_{p}^{2}=\sigma_{h}^{2}+\sigma_{z}^{2}=\sigma_{x}^{2}+\sigma_{y}^{2}+\sigma_{z}^{2} .
$$

Information about accuracy of coordinates data in ADS-B $\left(S_{\mathrm{ADS}-\mathrm{B}}\right)$ can be obtained directly from ADS-B message or can be estimated by math approach of GNSS error prediction for a particular point of airspace [21].

In our approach, we use information from ADS-B message. According to ADS-B data communication format, digital message should contain data about accuracy of positioning in the horizontal plane (NACp) (Navigation Accuracy Category for Position). The NACp value indicates the confidence band of data reported with a $95 \%$ probability level. Examples of NACp values with the correspondent confidence bands are given in Table 1 [9].

Also, performance of positioning can be improved by simple implementation of noise filtering at different levels of data processing [22].

Table 1. Navigation accuracy category for position data in ADS-B message format.

\begin{tabular}{|c|c|}
\hline NACp & The boundary of positioning error in the horizontal plane \\
\hline 0 & $\sigma_{\mathrm{p}} \geq 18.52 \mathrm{~km}(10 \mathrm{NM})$ \\
\hline 1 & $\sigma_{\mathrm{p}}<18.52 \mathrm{~km}(10 \mathrm{NM})$ \\
\hline 2 & $\sigma_{\mathrm{p}}<7.4 \mathrm{~km}(4 \mathrm{NM})$ \\
\hline 3 & $\sigma_{\mathrm{p}}<3.7 \mathrm{~km}(2 \mathrm{NM})$ \\
\hline 4 & $\sigma_{\mathrm{p}}<1852 \mathrm{~m}(1 \mathrm{NM})$ \\
\hline 5 & $\sigma_{\mathrm{p}}<926 \mathrm{~m}(0.5 \mathrm{NM})$ \\
\hline 6 & $\sigma_{\mathrm{p}}<555 \mathrm{~m}(0.3 \mathrm{NM})$ \\
\hline 7 & $\sigma_{\mathrm{p}}<185 \mathrm{~m}(0.1 \mathrm{NM})$ \\
\hline 8 & $\sigma_{\mathrm{p}}<92 \mathrm{~m}(0.05 \mathrm{NM})$ \\
\hline 9 & $\sigma_{\mathrm{p}}<30 \mathrm{~m}$ \\
\hline 10 & $\sigma_{\mathrm{p}}<10 \mathrm{~m}$ \\
\hline 11 & $\sigma_{\mathrm{p}}<3 \mathrm{~m}$ \\
\hline
\end{tabular}

\section{Numerical demonstration}

In a numerical demonstration, we use live air traffic data received by software defined radio. ADS-B receiver is located in $50.439490^{\circ} \mathrm{N}$ of latitude and $30.429708^{\circ} \mathrm{E}$ longitude. Data set is obtained in April 30, 2018 at 3:58 PM UTC. The decoded ADS-B 
messages were stored in separate matrices by unique airplane identifier. After recording air traffic reports for 30 minutes, the data of air traffic users locations was interpolated to a common time scale, because raw data from airplane transponders is not synchronized. Subsequently, for the simulation of the proposed method, an iterative approach was used in order to estimate the accuracy characteristics of the FL 195 for each point of the possible airplane location within the studied airspace volume. The results of availability estimation are presented in fig. 3. Obtained results indicates up to 13 airplanes available for positioning (marked by diamonds in fig. 3).

The accuracy of GNSS positioning is limited to a certain value for all airplanes within investigated airspace volume. Since the majority of errors depends on the state of the ionosphere, which varies simultaneously over a certain area. Therefore, it can be assumed that GNSS errors at a certain time and at the particular airspace have approximately the same probabilistic distribution.

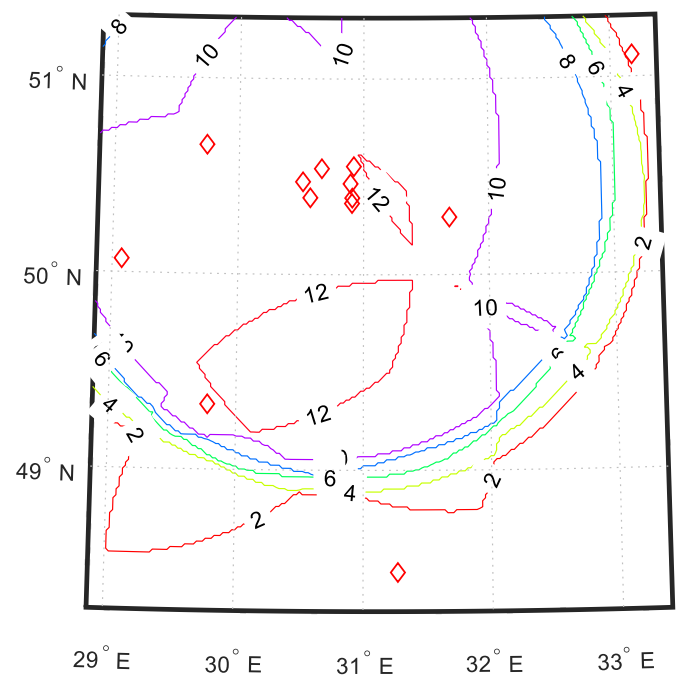

Fig. 3. Availability of ADS-B data for positioning.

Let's estimate the effect of errors, associated with uncertainty areas of ADS-B data, on the positioning results. To do this, we perform the estimation of $\sigma_{p}^{2}$ without taking into account uncertainty areas of airplane location by the weighted method. Similar to (11), we have:

$$
\left(H^{T} W^{-1} H\right)^{-1}=\left[\begin{array}{ccc}
\sigma_{x 0}^{2} & \sigma_{x y}^{2} & \sigma_{x z}^{2} \\
\sigma_{x y}^{2} & \sigma_{y 0}^{2} & \sigma_{y z}^{2} \\
\sigma_{x z}^{2} & \sigma_{y z}^{2} & \sigma_{z 0}^{2}
\end{array}\right]
$$

Positioning accuracy in the horizontal plane is:

$$
\sigma_{h 0}^{2}=\sigma_{x 0}^{2}+\sigma_{y 0}^{2}
$$

Total positioning accuracy in space is:

$$
\sigma_{p 0}^{2}=\sigma_{h 0}^{2}+\sigma_{z 0}^{2}=\sigma_{x 0}^{2}+\sigma_{y 0}^{2}+\sigma_{z 0}^{2}
$$

The effect of influence of uncertainty areas on the positioning results will be the error difference: 
- in horizontal plane: $\Delta \sigma^{2}{ }_{\mathrm{h}}=\left|\sigma_{\mathrm{h}}^{2}-\sigma^{2}{ }_{\mathrm{h} 0}\right|$;

- in vertical plane: $\Delta \sigma^{2}{ }_{\mathrm{z}}=\left|\sigma_{\mathrm{z}}^{2}-\sigma_{\mathrm{z} 0}^{2}\right|$;

- in total: $\Delta \sigma_{\mathrm{p}}^{2}=\left|\sigma_{\mathrm{p}}^{2}-\sigma_{\mathrm{p} 0}^{2}\right|$.

Values of $\Delta \sigma_{h}^{2}, \Delta \sigma_{z}^{2}$, and $\Delta \sigma_{p}^{2}$ utilizes affects the accuracy of positioning by performance of ADS-B data. Other important characteristic of positioning is horizontal dilution of precision coefficient (HDOP) that indicates relation of positioning error in horizontal plane to distance measurement error of ACAS surveillance sensor:

$$
H D O P=\sigma_{p l}^{2} / \sigma_{d}^{2}
$$

The results of HDOP estimation for the given geometry of air traffic are shown in fig. 4. According to classification [23], obtained HDOP values correspond to good performance level.

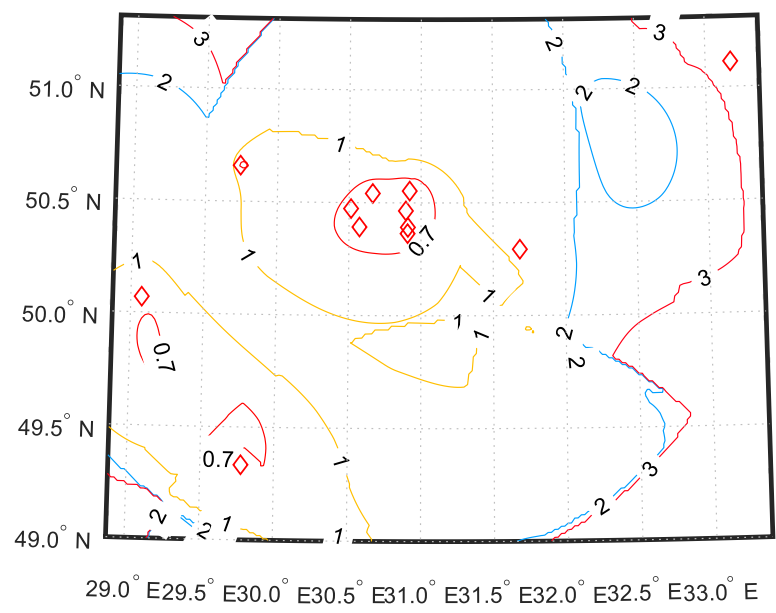

Fig. 4. HDOP coeficient.

Results of the estimation of positioning accuracy by (12) and (14) are presented in fig. 5 and fig. 6 corespondently. Difference (15) is presented in fig. 7.

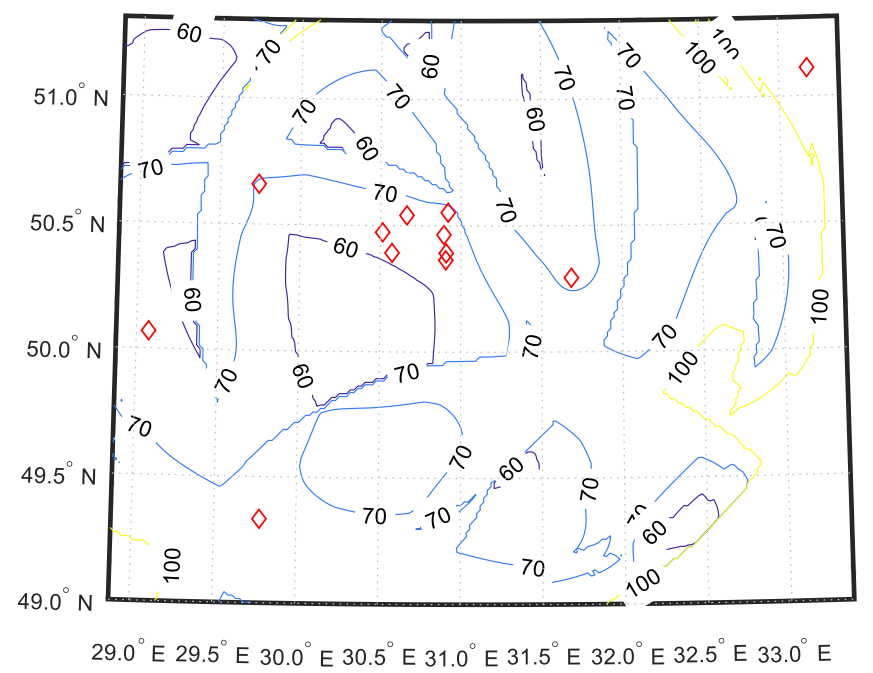

Fig. 5. Accuracy of positioning in horizontal plane with uncirtancies of navigation points location. 


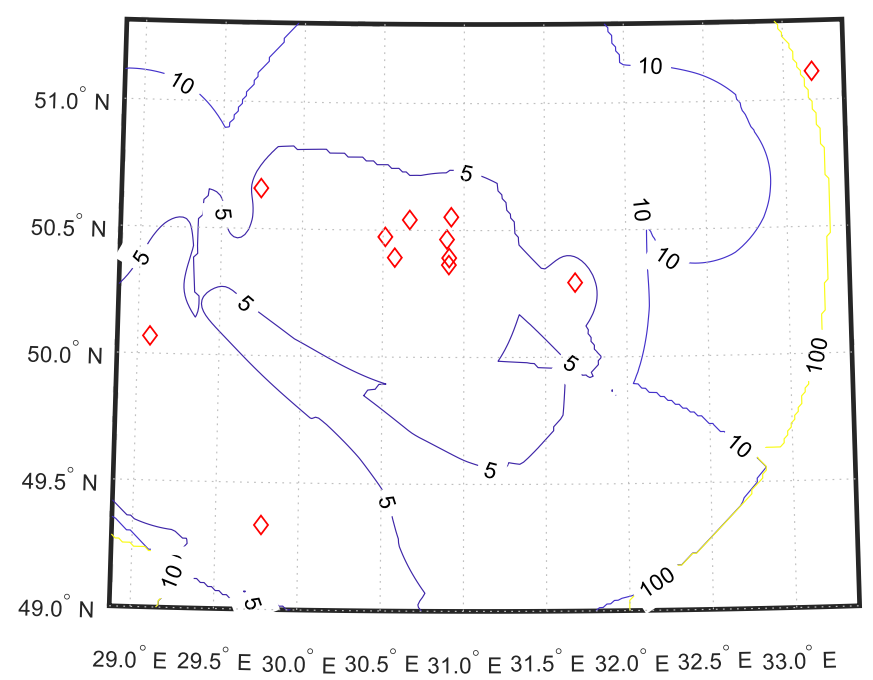

Fig. 6. Accuracy of positioning in horizontal plane by (14).

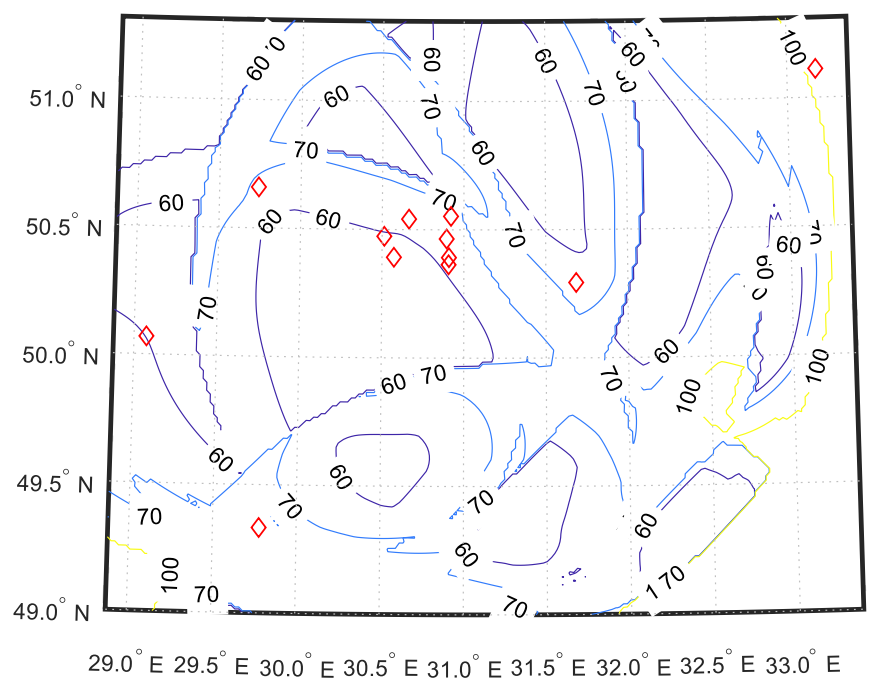

Fig. 7. Degradation of precision due to errors in ADS-B data.

Also, obtained results can be represented in values of area that support particular accuracy level. Results of availability estimation for particular values of $\sigma_{h}, \sigma_{h 0}$, and $\Delta \sigma_{h}$ are indicated in fig. 8. 


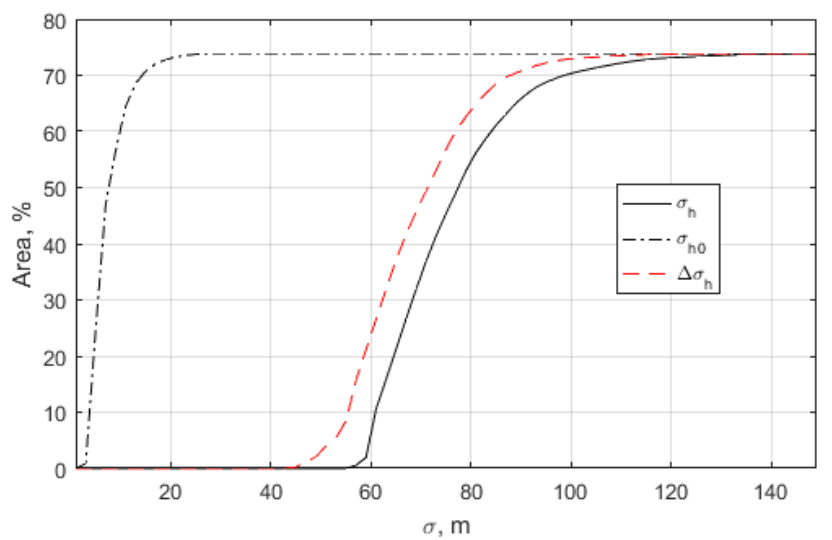

Fig. 8. Area estimation for particular values of $\sigma$.

Results in fig. 8 indicates possibility of getting airplane location with error less than $10 \mathrm{~m}$ for $73 \%$ of airspace in case of GNSS error negligee. But, in case of error influence positioning performance is reduced for $120 \mathrm{~m}$ for the same airspace volume.

\section{Conclusions}

An airplane coordinates detection is related to one of the most important navigation tasks, due to direct influence of availability, continuity, and accuracy of data on safety of aviation transport. Development of new version of collision avoidance system (ACAS X) opens a new challenge for surveillance and positioning in terms of relative navigation theory. Proposed new positioning method by surveillance and ADS-B data can be implemented in ACAS X as an alternative or stand-by positioning method on-board of airplane, that can be used in case of primary positioning system malfunction.

Obtained math equation (11) can be useful for accuracy estimation of proposed positioning approach that takes into account uncertainty areas of each navigation point obtained under support of ADS-B data.

Results of HDOP and accuracy estimation indicate high dependence from geometry of mutual air traffic location in space (fig. 4) and dependence from uncertainty areas built under the ADS-B data (fig. 7). According to obtained accuracy analysis, proposed positioning method is applicable in airspace with high capacity. Also, accuracy of proposed positioning method depends on a number of air space users in area of ACAS surveillance mode operation.

The works presented in this paper is a part of project "An Investigation and Development of Alternative Methods of Positioning and Navigation for Air Transport". It is supported by the Fulbright scholar Grant in School of Aeronautics and Astronautics at Purdue University. The authors would like to acknowledge prof. Karen Marais, from AAE for her efforts associated with this work.

\section{References}

1. ACAS Guide Airborne Collision Avoidance (Eurocontrol, 2017)

2. A. Kulik, K. Dergachev, Intelligent transport systems in aerospace engineering 32, 243-303 (2016). DOI: 10.1007/978-3-319-19150-8_8

3. Airborne Collision Avoidance System (ACAS) Manual, Doc 9863 (International Civil Aviation Organization, 2006) 
4. International Standards and Recommended Practices. Aeronautical Telecommunications. Surveillance and collision avoidance system, Annex 10 to the convention on International Civil Aviation 4 (International Civil Aviation Organization, 2007).

5. Roadmap for the integration of civil Remotely-Piloted Aircraft Systems into the European Aviation System. Final report (European RPAS Steering Group, 2013)

6. R. Chamlou, Integrated Communications, Navigation, and Surveillance Conference Proceedings 7(1), 7-12 (2010). https://doi.org/10.1109/ICNSURV.2010.5503327

7. J. Jeannin, K. Ghorbal, Y. Kouskoulas, R. Gardner, A. Schmidt, E. Zawadzki, A. Platzer, Proceedings of the International Conference on Embedded Software, 127-136 (2015). DOI: 10.1109/EMSOFT.2015.7318268

8. Advisory Circular airworthiness approval of ADS-B Out System, AC 20-165, (Federal Aviation Authority, 2010)

9. M. Kastelein, M.U. De Haag, AIAA/IEEE Digital Avionics Systems Conference - Proceedings, (2014). DOI: 10.1109/DASC.2014.6979522

10. T. Humphreys, Springer Handbook of Global Navigation Satellite Systems, 469-503 (2017). DOI: $10.1007 / 978-3-319-42928-1 \_16$

11. P. Truffer, M. Scaramuzza, M. Troller, M. Bertschi, The 30th International Technical Meeting of the Satellite Division of the Institute of Navigation 2, 1258-1266 (2017)

12. X. Yang, F. Chen, W. Liu, F. Wang, The 2019 2nd IEEE International Conference on Information Communication and Signal Processing, ICICSP 2019, 117-120 (2019). DOI: 10.1109/ICICSP48821.2019.8958508

13. O. Solomentsev, M. Zaliskyi, Proceedings of IEEE 5th International Conference on Methods and Systems of Navigation and Motion Control, 123-126 (2018). DOI: 10.1109/MSNMC.2018.8576306

14. Aviation Safety Reporting System. Database Online. NASA (2020). https://asrs.arc.nasa.gov/search/database.html

15. I.V. Ostroumov, N.S. Kuzmenko, K. Marais, Proceedings of IEEE 5th International Conference on Methods and Systems of Navigation and Motion Control, 90-93 (2018). DOI: 10.1109/MSNMC.2018.8576293

16. N.S. Kuzmenko, I.V. Ostroumov, Proceedings of IEEE 1st International Conference on System Analysis and Intelligent Computing, (2018). DOI: 10.1109/SAIC.2018.8516790

17. Performance-based Navigation (PBN) Manual, Doc 9613 (International Civil Aviation Organization, 2008)

18. I.V. Ostroumov, N.S. Kuzmenko, Telecommunications and Radio Engineering 77(3), 211-223 (2018). DOI: 10.1615/TelecomRadEng.v77.i3.30

19. I. Ostroumov, V. Kharchenko, N. Kuzmenko, Aviation 23(2), 36-42 (2019). DOI: 10.3846/aviation.2019.10302

20. A. Tian, D. Dong, D. Ning, C. Fu, Proceedings - 6th International Symposium on Computational Intelligence and Design 2, 16-19 (2013). DOI: 10.1109/ISCID.2013.119

21. G. Sun, C. Wang, International Conference on E-Product E-Service and E-Entertainment, (2010). DOI: 10.1109/ICEEE.2010.5661131

22. N.S. Kuzmenko, I.V. Ostroumov, V.P. Kharchenko, Signal Processing Symposium, 109-114 (2019). DOI: 10.1109/SPS.2019.8882072

23. M. Tahsin, S. Sultana, T. Reza, M. Hossam-E-Haider, The 2nd International Conference on Electrical Engineering and Information and Communication Technology (2015). DOI: 10.1109/ICEEICT.2015.7307445 